\title{
Agreement of novel hemodynamic imaging parameters for the acute and chronic stages of ischemic stroke: a matched-pair cohort study
}

\author{
*Martina Sebök, MD, ${ }^{1,2}$ Christiaan Hendrik Bas van Niftrik, MD, PhD, ${ }^{1,2}$ Susanne Wegener, MD, ${ }^{2,3}$ \\ Andreas Luft, MD, ${ }^{2,3}$ Luca Regli, MD, ${ }^{1,2}$ and Jorn Fierstra, MD, PhD ${ }^{1,2}$ \\ Departments of ${ }^{1}$ Neurosurgery and ${ }^{3}$ Neurology, and ${ }^{2}$ Clinical Neuroscience Center, University Hospital Zurich, University of \\ Zurich, Switzerland
}

\begin{abstract}
OBJECTIVE In symptomatic patients with cerebrovascular steno-occlusive disease, impaired blood oxygenation leveldependent cerebrovascular reactivity (BOLD-CVR) and increased flow velocity of the $\mathrm{P}_{2}$ segment of the posterior cerebral artery $\left(\mathrm{PCA}-\mathrm{P}_{2}\right)$ on transcranial Doppler (TCD) ultrasonography have been introduced as emerging clinical imaging parameters to identify patients at high risk for recurrent ischemic events. Since hemodynamic physiology differs between the acute and chronic stages of ischemic stroke, the authors sought to investigate whether those parameters have merit for both the acute and chronic stages of ischemic stroke.
\end{abstract}

METHODS From a prospective database, patients who underwent BOLD-CVR and TCD examinations in the acute stroke stage (< 10 days) were matched to patients in the chronic stroke stage ( $>3$ months). A linear regression analysis for both groups was performed between ipsilateral PCA-P ${ }_{2}$ systolic flow velocity and BOLD-CVR of the ipsilateral (affected) hemisphere, the ipsilateral middle cerebral artery (MCA) territory, and the ipsilateral steal volume (i.e., paradoxical BOLD-CVR response). The resulting slopes and intercepts were statistically compared to evaluate differences between groups.

RESULTS Forty matched patient pairs were included. Regression analysis showed no significant difference for either the intercept $(p=0.84)$ or the slope $(p=0.85)$ between PCA-P flow velocity and BOLD-CVR as measured for the ipsilateral (affected) hemisphere. Similarly, no significant difference was seen between PCA-P flow velocity and BOLD-CVR of the ipsilateral MCA territory (intercept, $p=0.72$; slope, $p=0.36$ ) or between $P C A-P_{2}$ flow velocity and steal volume (intercept, $p=0.59$; slope, $p=0.34$ ).

CONCLUSIONS The study results indicated that the relationship between ipsilateral PCA-P ${ }_{2}$ systolic flow velocity and BOLD-CVR remains the same during the acute and chronic stages of ischemic stroke. This provides further support that these novel hemodynamic imaging parameters may have merit to assess the risk for recurrent ischemic events for a wide ischemic stroke population. PCA- $\mathrm{P}_{2}$ systolic flow velocity, in particular, may be a highly practical screening tool, independent of ischemic stroke stage.

https://thejns.org/doi/abs/10.3171/2021.4.FOCUS21125

KEYWORDS BOLD-CVR; PCA-P flow; hemodynamic; steno-occlusive disease; stroke

$\mathrm{T}$ o advance ischemic stroke care, clinically versatile imaging parameters to identify patients with symptomatic cerebrovascular steno-occlusive disease at highest risk for recurrent stroke are strongly desired. ${ }^{1}$ Although increased oxygen extraction fraction from PET and impaired cerebrovascular reactivity (CVR) from xenon-CT and SPECT have been strongly associated with recurrent stroke risk, ${ }^{2-5}$ these imaging modalities are not feasible for routine clinical stroke imaging.
To address this well-known challenge, we have recently validated two emerging imaging parameters derived from existing clinical imaging techniques that have ample availability in routine ischemic stroke management in patients with symptomatic cerebrovascular steno-occlusive disease as follows:

1. Increased ipsilateral systolic flow of the $\mathrm{P}_{2}$ segment of the posterior cerebral artery (PCA- $\mathrm{P}_{2}$ ) from transcranial Doppler (TCD) ultrasonography. ${ }^{6,7}$ TCD ul-

ABBREVIATIONS BOLD = blood oxygenation level-dependent; CVR = cerebrovascular reactivity; ICA = internal carotid artery; MCA = middle cerebral artery; MP-RAGE = magnetization-prepared rapid acquisition gradient echo; $\mathrm{PCA}-\mathrm{P}_{2}=\mathrm{P}_{2}$ segment of the posterior cerebral artery; $T C D=$ transcranial Doppler.

SUBMITTED February 28, 2021. ACCEPTED April 7, 2021

INCLUDE WHEN CITING DOI: 10.3171/2021.4.FOCUS21125.

* M.S. and C.H.B.v.N. contributed equally to this work. 
trasonography is routinely used in the diagnosis and management of symptomatic steno-occlusive disease. ${ }^{8}$ Increased PCA- $\mathrm{P}_{2}$ systolic flow velocity is a surrogate marker for the necessity of leptomeningeal collateral activation to compensate a state of hypoperfusion ${ }^{6,9}$ and has been linked to increased risk for recurrent ischemia and increased steal volume (i.e., paradoxical CVR). ${ }^{6,10}$

2. CVR measured quantitatively with blood oxygenation level-dependent (BOLD) functional MRI during a standardized hypercapnic- $\mathrm{CO}_{2}-$ stimulus (BOLD-CVR) has demonstrated a good agreement with hemodynamic failure derived from acetazolamide-challenged $\left({ }^{15} \mathrm{O}-\right)$ $\mathrm{H}_{2} \mathrm{O}$-PET. ${ }^{7,11,12}$ CVR describes the remaining vasodilatory capacity at the brain tissue level, whereas impaired CVR, ${ }^{12-14}$ especially paradoxical BOLD-CVR (i.e., steal volume), has been associated with an increased risk for recurrent ischemic events. ${ }^{1,15,16}$

Recently, we have found a strong agreement between PCA-P $\mathrm{P}_{2}$ systolic flow velocity and BOLD-CVR, with higher PCA- $\mathrm{P}_{2}$ systolic flow velocity values correlating to impaired BOLD-CVR and steal volume.7,17 This makes PCA-P $\mathrm{P}_{2}$ flow velocity, which can even be performed at the bedside, ${ }^{8}$ eligible as a clinical screening tool for symptomatic steno-occlusive patients prone to ischemic stroke recurrence who are in need of a further hemodynamic workup to assess brain tissue perfusion, typically obtained with advanced neuroimaging techniques such as PET, SPECT, or BOLD-CVR.

Hemodynamic adaptations, such as collateral flow activation and compensatory autoregulation of the cerebrovascular reserve capacity, however, may follow a different pattern for various stages of ischemic stroke. ${ }^{14,18-20}$ Most studies investigating ischemic stroke hemodynamics interchangeably merge patient data from acute stages with chronic stages after ischemic stroke. Therefore, it is unknown whether increased ipsilateral PCA-P $\mathrm{P}_{2}$ flow activation and ipsilateral BOLD-CVR impairment have clinical merit for both the acute and chronic stages of ischemic stroke. ${ }^{7,12,17}$

Hence, our aim was to compare symptomatic patients with steno-occlusive disease who underwent BOLD-CVR and TCD ultrasonography in the acute stage of ischemic stroke ( $<10$ days) with those who underwent imaging in the chronic stage ( $>3$ months) using a matched-pair cohort study design.

\section{Methods \\ Patient Selection}

The data that support the findings of this study are available on reasonable request from the corresponding author (M.S.). This project is part of an ongoing BOLDCVR study in patients with symptomatic cerebrovascular steno-occlusive disease that has been approved by the local research ethics board (Kantonale Ethikkommission Zurich). Written informed consent was obtained from each participant before inclusion in the database. The study was conducted in accordance with the ethical standards as defined in the 1964 Declaration of Helsinki and its later amendments.

The inclusion criteria were 1) patient age 18 years or older with symptomatic unilateral steno-occlusive disease and 2) who exhibited focal neurological symptoms that were sudden in onset and referable to the appropriate anterior circulation large-artery distribution (ipsilateral to the significant large-vessel atherosclerotic pathology), including one or more transient ischemic attacks, characterized by focal neurological dysfunction or transient monocular blindness, or one or more minor (nondisabling) ischemic strokes. ${ }^{21}$ Unilateral disease was considered as a maximal stenosis of $50 \%$ on the contralateral side graded by duplex sonography according to the NASCET (North American Symptomatic Carotid Endarterectomy Trial) criteria. ${ }^{22}$

First, all symptomatic patients with unilateral stenoocclusive disease who underwent a separate clinical TCD ultrasonography investigation were selected from the database. For the acute group (scanning performed $<10$ days from the stroke event), only patients who underwent both investigations within 1 week were included in this study. For the chronic group (scanning performed $>90$ days from the stroke event), we allowed for an interval of 8 weeks, and TCD ultrasonography could not have been performed in the acute stroke phase. Patients with an insufficient insonation bone window by TCD examination, a vascular pathology of the posterior circulation, or bilateral anterior circulation steno-occlusive disease (inclusive of moyamoya disease) were excluded from further analysis.

After evaluation, 165 patients (96 with acute stroke and 69 with chronic stroke) qualified for inclusion. Based on the average BOLD-CVR values of the ipsilateral hemisphere, we were able to match 80 patients (40 per group) and include them for further analysis. Patients who underwent both BOLD-CVR and TCD ultrasonography studies in the acute stage of ischemic stroke ( $<10$ days) composed the acute hemodynamic cohort, whereas patients who underwent both examinations in the chronic stroke stage $(>3$ months) made up the chronic hemodynamic cohort. Some patient data from this cohort have been reported previously. ${ }^{7,17}$

\section{Image Acquisition and Analysis}

BOLD functional MRI images were obtained using a 3-T Skyra VD13 (Siemens Healthcare) scanner with $\mathrm{CO}_{2}$ as the vasoactive stimulus modulated by a computercontrolled gas blender with prospective gas-targeting algorithms (RespirAct, Thornhill Research Institute). This allowed for a short 80-second duration of iso-oxic hypercapnia to induce a vascular response. A high-resolution T1-weighted magnetization-prepared rapid acquisition gradient echo (MP-RAGE) image was obtained for anatomical overlay of the BOLD-CVR images. All BOLDCVR images were obtained using a previously published method, ${ }^{23}$ which has been used in multiple studies. ${ }^{14,17,20}$ BOLD-CVR, defined as the percentage of the BOLD signal change/mm $\mathrm{Hg} \mathrm{CO}$, was calculated from the slope of a linear least-square fit of the BOLD signal time course to the $\mathrm{CO}_{2}$ time course during the BOLD scan. ${ }^{23}$

From the T1-weighted MP-RAGE scan, a probability map for the gray matter, white matter, and CSF was obtained. Each T1-weighted MP-RAGE image was then manually masked for the affected hemisphere, and in combination with a gray-white matter probability map ( $>80 \%$ 


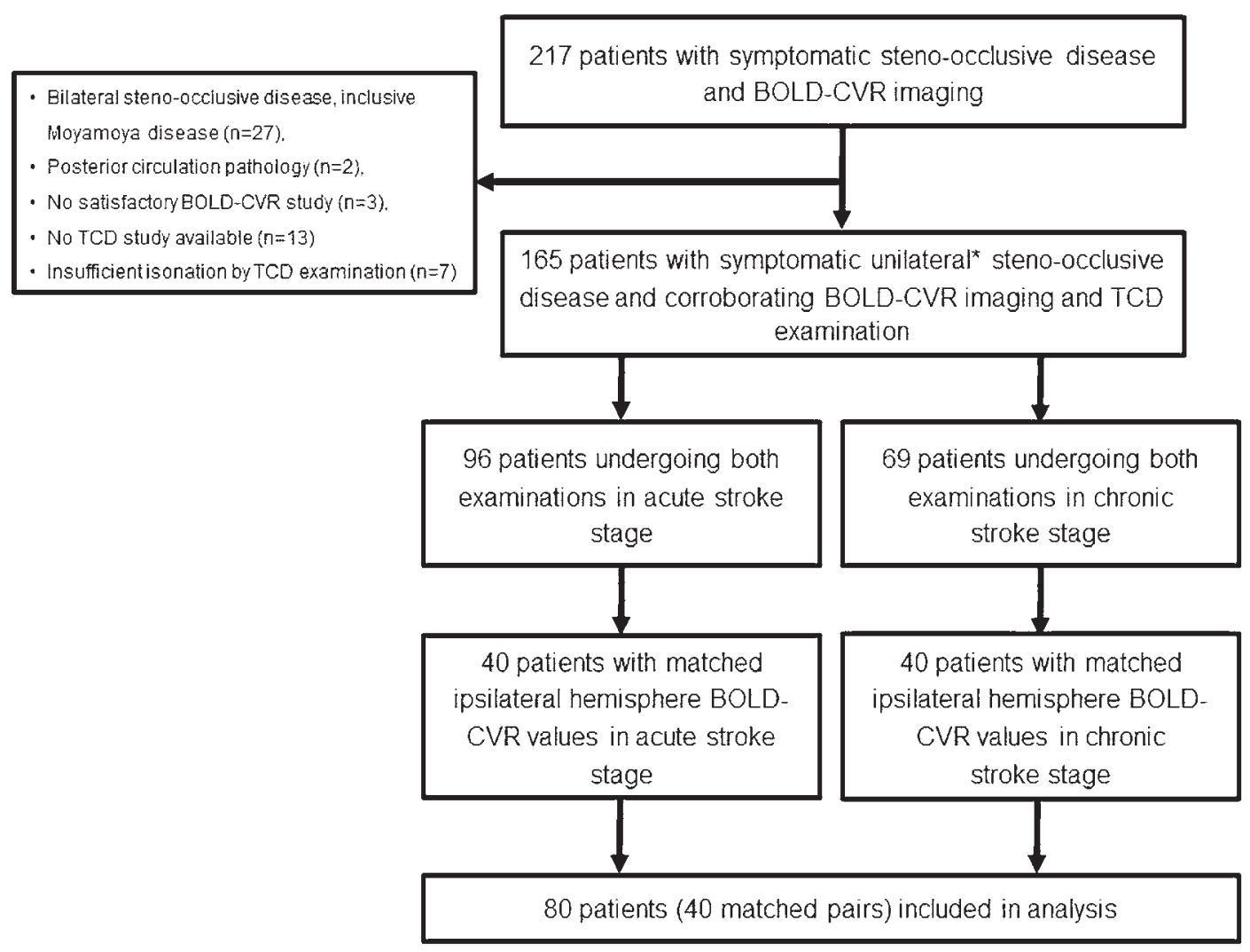

FIG. 1. Study flowchart. From 217 patients with symptomatic steno-occlusive disease included in our prospective BOLD-CVR database, 165 patients were diagnosed with unilateral steno-occlusive disease and had corroborating BOLD-CVR and TCD ultrasonography examination findings. Ninety-six of 165 patients underwent both examinations in the acute ischemic stroke stage, whereas 69 patients underwent both examinations in the chronic stroke stage. After a matching process, based on the ipsilateral hemisphere BOLD-CVR values, we were able to include 80 patients ( 40 per group). *Unilateral disease was considered as maximal stenosis of $50 \%$ of the contralateral side.

probability), CVR of the affected hemisphere was calculated.

\section{Steal Volume Analysis}

Steal volume, describing the number of voxels with paradoxical (i.e., negative) BOLD-CVR and measured in milliliters, was determined by measuring the number of voxels in the whole brain as well as in the ipsilateral and contralateral hemispheres, with $<0 \%$ BOLD signal change/mm $\mathrm{Hg} \mathrm{CO}$ after exclusion of voxels around the frontal sinus in order to exclude artifacts.

\section{Analysis of the MCA Territory}

Quantitative BOLD-CVR values of the middle cerebral artery (MCA) territory of the ipsilateral and contralateral hemispheres were determined by applying a vascular atlas to the normalized CVR maps. This vascular atlas was derived from the predefined brain regions listed in the standard N30R83 atlas by Hammers et al. ${ }^{24}$ and Kuhn et al. ${ }^{25}$

\section{Statistical Analysis}

Statistical analysis was performed using IBM SPSS Statistics version 26 (IBM Corp.). All normally distributed continuous variables are reported as mean \pm SD. Cat- egorical ordinal variables are presented as median (IQR), whereas dichotomous variables are shown as frequency (\%). To evaluate the comparability of both groups, the chisquare test and the Student t-test were used. A 2-sided $\mathrm{p}$ value $<0.05$ was considered significant. A linear regression analysis for both groups was performed between the ipsilateral PCA- $\mathrm{P}_{2}$ systolic flow velocity and BOLD-CVR of the ipsilateral hemisphere, and BOLD-CVR of the ipsilateral MCA territory and the ipsilateral steal volume. The resulting slopes and intercepts were statistically compared to evaluate differences between groups.

\section{Results}

\section{Study Population Characteristics}

A flowchart illustrating patient screening and inclusion is shown in Fig. 1. In the acute hemodynamic cohort, which included patients in an acute ischemic stroke stage, 20 patients with internal carotid artery (ICA) occlusion, 1 patient with MCA occlusion, 4 patients with tandem (ICA and MCA) occlusion, 13 patients with ICA stenosis, and 2 patients with MCA stenosis were included. The chronic hemodynamic cohort consisted of 23 patients with ICA occlusion, 1 patient with MCA occlusion, 15 patients with ICA stenosis, and 1 patient with MCA stenosis. 
TABLE 1. Baseline characteristics of included symptomatic steno-occlusive patients

\begin{tabular}{|c|c|c|c|c|}
\hline & All Patients $(n=80)$ & Acute Cohort $(n=40)$ & Chronic Cohort $(n=40)$ & $p$ Value \\
\hline Mean age, yrs & $67.89 \pm 10.41$ & $69.51 \pm 10.90$ & $66.26 \pm 9.77$ & 0.16 \\
\hline Male sex & $66(82.5)$ & $31(77.5)$ & $35(87.5)$ & 0.25 \\
\hline Mean vol of ischemic lesions, $\mathrm{ml}$ & $10.81 \pm 23.37$ & $12.00 \pm 17.70$ & $9.61 \pm 28.11$ & 0.65 \\
\hline Smoking & $43(53.8)$ & $16(40)$ & $27(67.5)$ & 0.01 \\
\hline Hypertension & $51(63.7)$ & $26(65.0)$ & $25(62.5)$ & 0.82 \\
\hline Hypercholesterolemia & $36(45)$ & $20(50)$ & $16(40)$ & 0.38 \\
\hline Obesity & $14(17.5)$ & $10(25)$ & $4(10)$ & 0.08 \\
\hline Diabetes & $12(15)$ & $5(12.5)$ & $7(17.5)$ & 0.54 \\
\hline Positive family history for cerebral ischemic events & $8(10)$ & $2(5)$ & $6(15)$ & 0.14 \\
\hline
\end{tabular}

Values represent the number of patients (\%) unless stated otherwise. Mean values are presented as the mean \pm SD. Boldface type indicates statistical significance.

Table 1 shows the relevant clinical and baseline characteristics of the enrolled patients. As shown in Table 2, no significant differences were found in relevant BOLDCVR values of the whole brain, hemispheres, MCA territories, steal volumes, or PCA- $\mathrm{P}_{2}$ systolic flow velocities.

\section{Linear Regression Analysis for the Acute and Chronic Hemodynamic Cohorts}

PCA-P ${ }_{2}$ Systolic Flow Velocity and Ipsilateral Hemisphere BOLD-CVR

The linear regression curve between the quantitative ipsilateral PCA- $\mathrm{P}_{2}$ systolic flow velocity values and the mean ipsilateral hemisphere BOLD-CVR values showed

TABLE 2. BOLD-CVR and TCD values of acute and chronic symptomatic steno-occlusive patient cohorts

\begin{tabular}{|c|c|c|c|}
\hline & $\begin{array}{l}\text { Acute Cohort } \\
\quad(n=40)\end{array}$ & $\begin{array}{l}\text { Chronic Cohort } \\
\qquad(n=40)\end{array}$ & $\begin{array}{c}p \\
\text { Value }\end{array}$ \\
\hline \multicolumn{4}{|l|}{$\begin{array}{l}\text { Mean CVR, \% BOLD/mm } \\
\mathrm{Hg} \mathrm{CO}_{2}\end{array}$} \\
\hline Whole brain & $0.13 \pm 0.06$ & $0.14 \pm 0.06$ & 0.74 \\
\hline Gray matter & $0.17 \pm 0.11$ & $0.16 \pm 0.06$ & 0.54 \\
\hline White matter & $0.10 \pm 0.05$ & $0.10 \pm 0.05$ & 0.89 \\
\hline Contralat hemisphere & $0.15 \pm 0.06$ & $0.16 \pm 0.05$ & 0.72 \\
\hline Ipsilat MCA territory & $0.08 \pm 0.08$ & $0.10 \pm 0.08$ & 0.39 \\
\hline Contralat MCA territory & $0.15 \pm 0.06$ & $0.16 \pm 0.05$ & 0.31 \\
\hline \multicolumn{4}{|l|}{ Mean steal vol, ml } \\
\hline Whole brain & $109.00 \pm 113.32$ & $86.42 \pm 115.49$ & 0.38 \\
\hline Ipsilat hemisphere & $70.40 \pm 69.28$ & $54.73 \pm 76.36$ & 0.34 \\
\hline Contralat hemisphere & $38.61 \pm 58.75$ & $31.69 \pm 45.21$ & 0.56 \\
\hline \multicolumn{4}{|l|}{$\begin{array}{l}\text { Median SFV of PCA-P }, \\
\mathrm{cm} / \mathrm{sec}\end{array}$} \\
\hline Ipsilat & $57(34)$ & $52(33)$ & 0.70 \\
\hline Contralat & $55(28)$ & $51(30)$ & 0.85 \\
\hline
\end{tabular}

a moderate association between PCA- $\mathrm{P}_{2}$ flow velocity and BOLD-CVR for the acute phase $\left(\mathrm{R}=-0.53, \mathrm{R}^{2}=0.28, \mathrm{p}\right.$ $<0.001)$ and a good correlation for the chronic phase $(\mathrm{R}=$ $-0.60, R^{2}=0.36, p<0.001$ ). Regression analysis (Fig. 2A) showed no significant difference for either the intercept $(\mathrm{p}$ $=0.84)$ or the slope $(\mathrm{p}=0.85)$, indicating that a difference in the poststroke phase does not change the relationship between PCA-P ${ }_{2}$ flow velocity and BOLD-CVR as measured for the ipsilateral (affected) hemisphere.

\section{PCA-P $P_{2}$ Systolic Flow Velocity and Ipsilateral MCA Territory BOLD-CVR}

Similarly, the linear regression curve between the quantitative ipsilateral PCA- $\mathrm{P}_{2}$ systolic flow velocity values and the mean ipsilateral (affected) MCA territory BOLDCVR values showed a strong association in the acute $(\mathrm{R}=$ $\left.-0.61, \mathrm{R}^{2}=0.36, \mathrm{p}<0.001\right)$ and chronic $\left(\mathrm{R}=-0.54, \mathrm{R}^{2}=\right.$ $0.29, \mathrm{p}<0.001)$ stroke phases. Regression analysis showed that the stroke phase had no influence on this relationship (intercept, $\mathrm{p}=0.72$; slope, $\mathrm{p}=0.36$ ) (Fig. 2B).

\section{PCA- $\mathrm{P}_{2}$ Systolic Flow Velocity and Affected Hemisphere Steal Volume}

Lastly, we tested the relationship between the quantitative ipsilateral PCA- $\mathrm{P}_{2}$ systolic flow velocity values and the steal volume of the ipsilateral (affected) hemisphere. Both the acute and chronic stages, separately, showed a significant correlation (acute cohort: $\mathrm{R}=-0.39, \mathrm{R}^{2}=0.15$, $\mathrm{p}=0.013$; and chronic cohort: $\mathrm{R}=-0.64, \mathrm{R}^{2}=0.40, \mathrm{p}<$ 0.001 ). The correlation for the chronic stroke phase was not significantly different than the acute stroke phase (intercept, $\mathrm{p}=0.59$; slope, $\mathrm{p}=0.34)($ Fig. $2 \mathrm{C})$.

\section{Discussion}

The current matched-pair cohort study shows that the relationship between ipsilateral PCA- $\mathrm{P}_{2}$ systolic flow velocity and BOLD-CVR does not change for the acute and chronic stages of ischemic stroke. This may have clinical merit for a wide stroke population in order to identify symptomatic steno-occlusive patients at risk for recurrent ischemic events. Increased ipsilateral systolic PCA- $\mathrm{P}_{2}$ flow velocity, in particular, has been shown to independently correlate with impaired BOLD-CVR ${ }^{7}$ and steal volume ${ }^{17}$ 

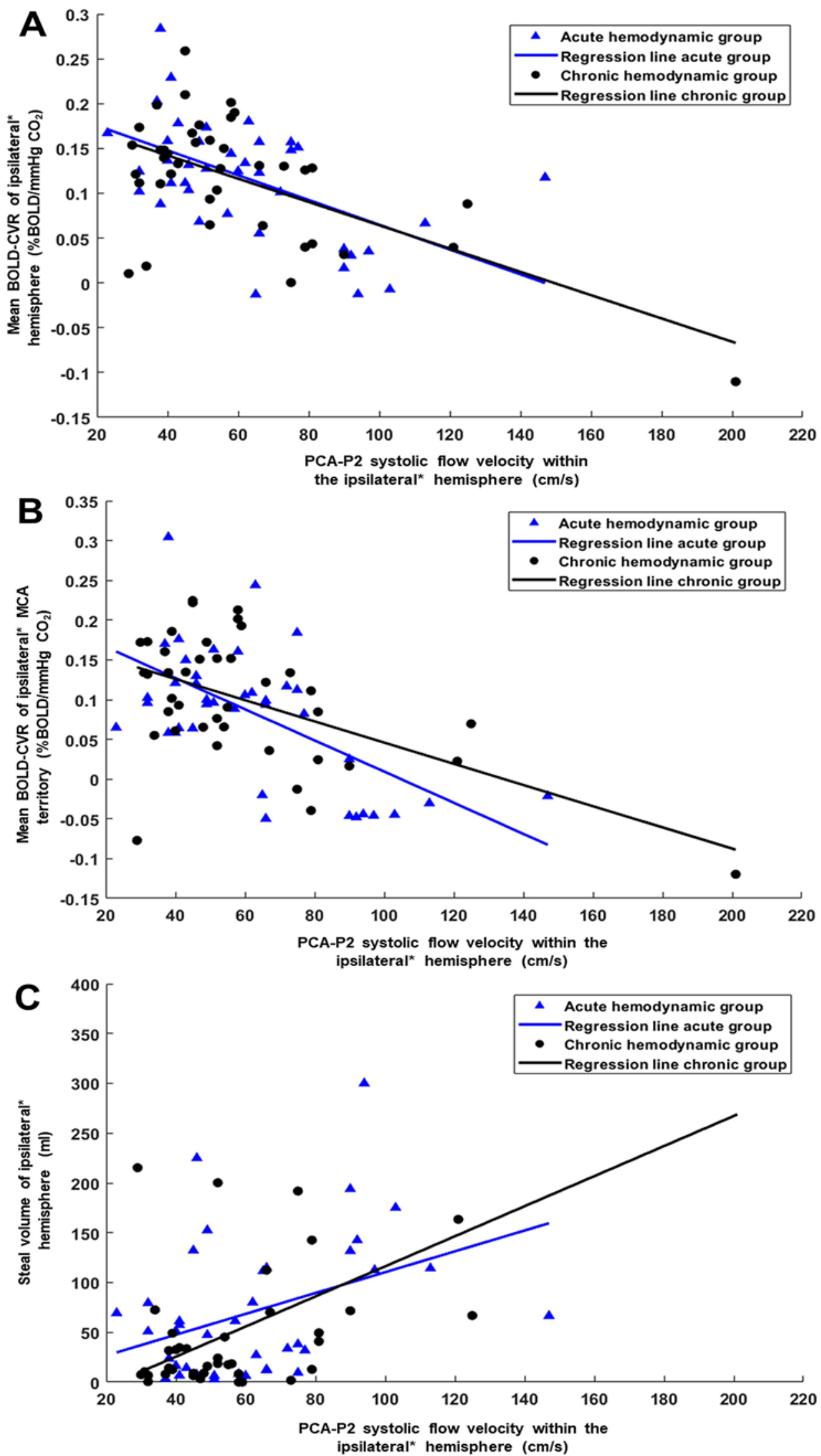

FIG. 2. Regression analysis of PCA-P $P_{2}$ systolic flow velocity and different BOLD-CVR parameters. A: Regression analysis showing no significant difference for both the intercept $(p=0.84)$ and slope $(p=0.85)$, indicating that the difference in the poststroke phase does not change the relationship between ipsilateral PCA-P ${ }_{2}$ systolic flow velocity and BOLD-CVR of the ipsilateral hemisphere. FIG. 2. (continued) $\rightarrow$ 
FIG. 2. B: Regression analysis showing no influence (intercept, $p=0.72$; slope, $p=0.36$ ) of the stroke phase on the relationship between ipsilateral PCA- $P_{2}$ systolic flow velocity and BOLD-CVR of the ipsilateral MCA territory. C: Regression analysis showing no significant difference (intercept, $p=$ 0.59 ; slope, $p=0.34$ ) between the acute and chronic hemodynamic groups for the relationship between ipsilateral PCA- $P_{2}$ systolic flow velocity and steal volume of the ipsilateral hemisphere. ${ }^{*}$ The ipsilateral hemisphere is considered the hemisphere on the side of the symptomatic steno-occlusive disease.

and is increased in patients with recurrent stroke. ${ }^{6}$ This association describes the necessity of leptomeningeal activation to compensate for a state of hypoperfusion, ${ }^{6,9}$ and makes PCA- $\mathrm{P}_{2}$ systolic flow velocity an ideal screening method for stroke patients requiring further hemodynamic workup. BOLD-CVR, the measurement of intracranial hemodynamic status at the brain tissue level, is a quantitative and highly reproducible imaging method and has shown a good agreement with CVR as measured using the clinical gold-standard $\left({ }^{15} \mathrm{O}-\right) \mathrm{H}_{2} \mathrm{O}$-PET examination. ${ }^{12}$ To allow for an optimal investigation of the relationship between PCA$\mathrm{P}_{2}$ flow velocities and BOLD-CVR during different stroke stages, patients were matched based on the mean BOLDCVR of the ipsilateral hemisphere. We found that the systolic PCA-P $\mathrm{P}_{2}$ flow velocity of the ipsilateral hemisphere did correlate during both the acute and chronic stroke stages with different BOLD-CVR measurements (ipsilateral hemisphere BOLD-CVR, BOLD-CVR of the ipsilateral MCA territory, and volume of the region with the steal phenomenon [i.e., paradoxical BOLD-CVR]). Moreover, no differences in the intercept and slope between each of the correlations were found, meaning that the influence of the stroke phase (acute vs chronic) on this relationship has been indiscernible. This indicates that systolic PCA- ${ }_{2}$ flow velocity can be used as an independent hemodynamic parameter in both acute and chronic hemodynamic stages in patients with symptomatic unilateral anterior circulation steno-occlusive disease.

\section{Hemodynamic Features of Anterior Circulation Steno-Occlusive Disease}

Atherosclerotic steno-occlusive disease describes a vascular state in which the extracranial or intracranial arteries supplying the brain show either a stenosis (abnormal narrowing of a blood vessel) or an occlusion (complete blockage of a blood vessel). A stenosis or occlusion of the appropriate anterior circulation large artery is an important cause of anterior circulation ischemic stroke and stroke recurrence and has been linked to chronic deficiencies in regional cerebral blood flow. ${ }^{26,27}$

Acute ischemia results in irreversible loss of brain tissue and function, ${ }^{28}$ whereas the presence of a chronic cerebrovascular steno-occlusive disease can alter cerebral hemodynamics up to a point where brain tissue perfusion becomes insufficient (chronic hypoperfusion). ${ }^{11,29-31}$ The consequences of chronic intermittent hypoperfusion are still not completely understood. It is supposed that the perfusion of brain tissue may be just sufficient enough to prevent gross ischemia but may fail to respond adequately to increases in demand such as those normally seen during neuronal activation. ${ }^{1,27}$ If hemodynamic impairment is present, it is hypothesized to alter brain structure and function..$^{27,32}$ Between acute and chronic stages of stroke, an evolution in collateral flow occurs either parallel to or as a response to these changes; ${ }^{18,33}$ therefore, the efficacy of hemodynamic parameters should be tested for both the acute and chronic stages of stroke. Such studies are lacking, as advanced hemodynamic imaging studies with PET or SPECT are usually difficult and expensive to obtain in a large cohort of stroke patients with varying stroke stages.

\section{Hemodynamic Impairment and Its Correlation With PCA-P ${ }_{2}$ Flow Velocity}

Previous studies highlighted the importance of systolic PCA- $\mathrm{P}_{2}$ flow velocity, as measured by TCD, as an independent marker of intracranial hemodynamic status in patients with steno-occlusive disease.$^{6,17}$ Since TCD is a noninvasive bedside method, it can be repeatedly used to evaluate the flow velocity of intra- and extracranial vessels, as well as the presence of primary and secondary collaterals, without the need for an exogenous contrast agent. ${ }^{8,33}$ A recent study showed that in patients with symptomatic unilateral steno-occlusive disease, increased ipsilateral TCD PCA- $\mathrm{P}_{2}$ systolic flow velocity is a strong independent predictor of hemodynamic impairment in the ipsilateral hemisphere and MCA territory. ${ }^{7}$ A flow increase $>30 \%$ in the ipsilateral PCA- $\mathrm{P}_{2}$ segment compared with the contralateral PCA- $\mathrm{P}_{2}$ segment indicates leptomeningeal collateral flow, known as a secondary collateral pathway. ${ }^{6,9,34}$ Such pathways are recruited once primary collaterals ${ }^{19,35}$ have failed to sufficiently compensate, indicating a more severe impairment. ${ }^{10,36}$ Therefore, a necessary activation of secondary collaterals (e.g., leptomeningeal pial branches over the posterior circulation) has been associated with an increased risk of recurrent stroke. ${ }^{6,10,18}$

Using the systolic PCA-P $\mathrm{P}_{2}$ flow velocity of the ipsilateral hemisphere, we have found a significant correlation between different quantitative BOLD-CVR measurements on the brain tissue level without an influence of the hemodynamic stage (acute vs chronic) on this correlation. The positive correlation between PCA- $\mathrm{P}_{2}$ flow velocity and steal volume is especially interesting. Steal volume (i.e., a paradoxical, negative BOLD-CVR response to hypercapnia) is a prime hemodynamic parameter or hemodynamic failure type $2^{12}$ and describes the classic state of hypoperfusion (i.e., tissue at risk for recurrent ischemic stroke). ${ }^{37}$ This indicates that despite hemodynamic changes over time, PCA- $\mathrm{P}_{2}$ flow velocity remains linked to the present hemodynamic state of patients with anterior circulation steno-occlusive disease.

\section{Clinical Implications and Future Direction}

The good agreement found between increased ipsilateral $\mathrm{P}^{2}$ TCD flow velocity and impaired BOLD-CVR for the acute and chronic stages of ischemic stroke has a potentially important clinical application for a wide stroke population, since both novel parameters have individually been associated with hemodynamic failure ${ }^{6,7,12}$ (i.e., the imaging parameter correlated with recurrent ischemic stroke $^{1,15,16}$ ).

Furthermore, this study has underlined the value of 
TCD ultrasonography in combination with a quantifiable hemodynamic parameter (i.e., increased ipsilateral $\mathrm{P}_{2}$ flow velocity) as a screening tool for a wide stroke population in order to identify patients with potential hemodynamic failure ${ }^{6,7}$ that will need further imaging workup with either BOLD-CVR, acetazolamide-challenged $\mathrm{H}_{2} \mathrm{O}$-PET, SPECT, MR perfusion, or whichever modality is considered the institutional clinical standard to assess brain tissue perfusion.

Finally, although randomized controlled clinical trials $^{38,39}$ have failed to show a benefit of cerebral bypass revascularization for stroke prevention, routine clinical experience has indicated that a subset of patients with both acute and chronic ischemic stroke exhibiting impaired or even exhausted cerebral hemodynamic status could benefit from surgical or endovascular recanalization procedures. ${ }^{4-42}$ To detect at-risk patients requiring further and more complex hemodynamic investigations, a noninvasive and bedside-available TCD ultrasonography-derived systolic PCA-P ${ }_{2}$ flow velocity can be widely implemented as an additional triage investigation. ${ }^{8}$

\section{Limitations}

The inclusion of patients with different unilateral symptomatic anterior circulation ischemic stroke pathology may have influenced our findings. Specifically, our study enrolled a mixed cohort of symptomatic patients with steno-occlusive disease; that is, patients with occlusion as well as stenosis were included. Cerebral autoregulation was reported to worsen between the acute and subacute stroke phase; therefore, this may have influenced our results, as BOLD-CVR and TCD ultrasonography investigations were not always done on the same day. ${ }^{43,44}$

\section{Conclusions}

Our study indicates that the relationship between ipsilateral PCA- $\mathrm{P}_{2}$ systolic flow velocity and BOLD-CVR does not change for the acute and chronic stages of ischemic stroke. This provides further support that these novel hemodynamic imaging parameters may have merit to assess the risk for recurrent ischemic events for a wide ischemic stroke population. PCA-P $\mathrm{P}_{2}$ systolic flow velocity, in particular, may be a highly practical screening tool, independent of ischemic stroke stage.

\section{Acknowledgments}

This project was funded by the Clinical Research Priority Program of the University of Zurich (UZH CRPP Stroke). Dr. Susanne Wegener received funding from the Swiss National Science Foundation (SNSF PP00P3_170683).

\section{References}

1. Yonas H, Smith HA, Durham SR, et al. Increased stroke risk predicted by compromised cerebral blood flow reactivity. $J$ Neurosurg. 1993;79(4):483-489.

2. Markus H, Cullinane M. Severely impaired cerebrovascular reactivity predicts stroke and TIA risk in patients with carotid artery stenosis and occlusion. Brain. 2001;124(pt 3): 457-467.

3. Webster MW, Makaroun MS, Steed DL, et al. Compromised cerebral blood flow reactivity is a predictor of stroke in patients with symptomatic carotid artery occlusive disease. $J$ Vasc Surg. 1995;21(2):338-345.

4. Powers WJ, Zazulia AR. PET in cerebrovascular disease. PET Clin. 2010;5(1):83-106.

5. Grubb RL Jr, Powers WJ, Clarke WR, et al. Surgical results of the Carotid Occlusion Surgery Study. J Neurosurg. 2013; 118(1):25-33.

6. Schneider J, Sick B, Luft AR, Wegener S. Ultrasound and clinical predictors of recurrent ischemia in symptomatic internal carotid artery occlusion. Stroke. 2015;46(11): 3274-3276.

7. van Niftrik CHB, Sebök M, Wegener S, et al. Increased ipsilateral posterior cerebral artery $\mathrm{P} 2$-segment flow velocity predicts hemodynamic impairment. Stroke. 2021;52: 1469-1472.

8. Bonow RH, Young CC, Bass DI, et al. Transcranial Doppler ultrasonography in neurological surgery and neurocritical care. Neurosurg Focus. 2019;47(6):E2.

9. Reinhard M, Müller T, Guschlbauer B, et al. Dynamic cerebral autoregulation and collateral flow patterns in patients with severe carotid stenosis or occlusion. Ultrasound Med Biol. 2003;29(8):1105-1113.

10. Klijn CJ, Kappelle LJ, van Huffelen AC, et al. Recurrent ischemia in symptomatic carotid occlusion: prognostic value of hemodynamic factors. Neurology. 2000;55(12):1806-1812.

11. Fierstra J, Poublanc J, Han JS, et al. Steal physiology is spatially associated with cortical thinning. J Neurol Neurosurg Psychiatry. 2010;81(3):290-293.

12. Fierstra J, van Niftrik C, Warnock G, et al. Staging hemodynamic failure with blood oxygen-level-dependent functional magnetic resonance imaging cerebrovascular reactivity: a comparison versus gold standard $\left({ }^{15} \mathrm{O}-\right) \mathrm{H}_{2} \mathrm{O}$-positron emission tomography. Stroke. 2018;49(3):621-629.

13. Mandell DM, Han JS, Poublanc J, et al. Mapping cerebrovascular reactivity using blood oxygen level-dependent MRI in Patients with arterial steno-occlusive disease: comparison with arterial spin labeling MRI. Stroke. 2008;39(7):20212028.

14. Sebök M, van Niftrik CHB, Piccirelli M, et al. BOLD cerebrovascular reactivity as a novel marker for crossed cerebellar diaschisis. Neurology. 2018;91(14):e1328-e1337.

15. Silvestrini M, Vernieri F, Pasqualetti $P$, et al. Impaired cerebral vasoreactivity and risk of stroke in patients with asymptomatic carotid artery stenosis. JAMA. 2000;283(16): 2122-2127.

16. Schoof J, Lubahn W, Baeumer M, et al. Impaired cerebral autoregulation distal to carotid stenosis/occlusion is associated with increased risk of stroke at cardiac surgery with cardiopulmonary bypass. J Thorac Cardiovasc Surg. 2007; 134(3):690-696.

17. von Bieberstein L, van Niftrik CHB, Sebök M, et al. Crossed cerebellar diaschisis indicates hemodynamic compromise in ischemic stroke patients. Transl Stroke Res. 2021;12(1): 39-48.

18. Klijn CJ, Kappelle LJ. Haemodynamic stroke: clinical features, prognosis, and management. Lancet Neurol. 2010; 9(10):1008-1017.

19. van Everdingen KJ, Visser GH, Klijn CJ, et al. Role of collateral flow on cerebral hemodynamics in patients with unilateral internal carotid artery occlusion. Ann Neurol. 1998; 44(2):167-176.

20. Hendrik Bas van Niftrik C, Sebok M, Muscas G, et al. Characterizing ipsilateral thalamic diaschisis in symptomatic cerebrovascular steno-occlusive patients. J Cereb Blood Flow Metab. 2020;40(3):563-573.

21. Barnett HJM, Taylor DW, Haynes RB, et al. Beneficial effect of carotid endarterectomy in symptomatic patients with highgrade carotid stenosis. N Engl J Med. 1991;325(7):445-453. 
22. North American Symptomatic Carotid Endarterectomy Trial. Methods, patient characteristics, and progress. Stroke. 1991; 22(6):711-720.

23. van Niftrik $\mathrm{CHB}$, Piccirelli $\mathrm{M}$, Bozinov $\mathrm{O}$, et al. Iterative analysis of cerebrovascular reactivity dynamic response by temporal decomposition. Brain Behav. 2017;7(9):e00705.

24. Hammers A, Allom R, Koepp MJ, et al. Three-dimensional maximum probability atlas of the human brain, with particular reference to the temporal lobe. Hum Brain Mapp. 2003; 19(4):224-247.

25. Kuhn FP, Warnock G, Schweingruber T, et al. Quantitative $\mathrm{H} 2[(15) \mathrm{O}]-\mathrm{PET}$ in pediatric moyamoya disease: evaluating perfusion before and after cerebral revascularization. $J$ Stroke Cerebrovasc Dis. 2015;24(5):965-971.

26. Arenillas JF. Intracranial atherosclerosis: current concepts. Stroke. 2011;42(1)(suppl):S20-S23.

27. Fierstra J, Maclean DB, Fisher JA, et al. Surgical revascularization reverses cerebral cortical thinning in patients with severe cerebrovascular steno-occlusive disease. Stroke. 2011 42(6):1631-1637.

28. Desai SM, Rocha M, Jovin TG, Jadhav AP. High variability in neuronal loss. Stroke. 2019;50(1):34-37.

29. Sekhon LH, Morgan MK, Spence I, Weber NC. Chronic cerebral hypoperfusion and impaired neuronal function in rats. Stroke. 1994;25(5):1022-1027.

30. Muller M, van der Graaf Y, Algra A, et al. Carotid atherosclerosis and progression of brain atrophy: the SMART-MR study. Ann Neurol. 2011;70(2):237-244.

31. Bakker FC, Klijn CJ, Jennekens-Schinkel A, et al. Cognitive impairment in patients with carotid artery occlusion and ipsilateral transient ischemic attacks. J Neurol. 2003;250(11): $1340-1347$.

32. Jussen D, Zdunczyk A, Schmidt S, et al. Motor plasticity after extra-intracranial bypass surgery in occlusive cerebrovascular disease. Neurology. 2016;87(1):27-35.

33. Liebeskind DS. Collaterals in acute stroke: beyond the clot. Neuroimaging Clin N Am. 2005;15(3):553-573, x.

34. Baumgartner RW, Baumgartner I, Mattle HP, Schroth G. Transcranial color-coded duplex sonography in unilateral flow-restrictive extracranial carotid artery disease. AJNR Am J Neuroradiol. 1996;17(4):777-783.

35. Badacz R, Przewlocki T, Karch I, et al. Low prevalence of collateral cerebral circulation in the circle of Willis in patients with severe carotid artery stenosis and recent ischemic stroke. Postep Kardiol Inter. 2015;11(4):312-317.

36. Liebeskind DS. Collateral circulation. Stroke. 2003;34(9): 2279-2284.

37. Kataoka H, Miyamoto S, Ogasawara K, et al. Results of prospective cohort study on symptomatic cerebrovascular occlusive disease showing mild hemodynamic compromise [Japanese Extracranial-Intracranial Bypass Trial (JET)-2 Study]. Neurol Med Chir (Tokyo). 2015;55(6):460-468.
38. Powers WJ, Clarke WR, Grubb RL Jr, et al. Extracranialintracranial bypass surgery for stroke prevention in hemodynamic cerebral ischemia: the Carotid Occlusion Surgery Study randomized trial. JAMA. 2011;306(18):1983-1992.

39. EC/IC Bypass Study Group. Failure of extracranial-intracranial arterial bypass to reduce the risk of ischemic stroke. Results of an international randomized trial. $N$ Engl J Med. 1985;313(19):1191-1200.

40. Burkhardt JK, Winklhofer S, Fierstra J, et al. Emergency extracranial-intracranial bypass to revascularize salvageable brain tissue in acute ischemic stroke patients. World Neurosurg. 2018;109:e476-e485.

41. Nussbaum ES, Erickson DL. Extracranial-intracranial bypass for ischemic cerebrovascular disease refractory to maximal medical therapy. Neurosurgery. 2000;46(1):37-43.

42. Nussbaum ES, Janjua TM, Defillo A, et al. Emergency extracranial-intracranial bypass surgery for acute ischemic stroke. J Neurosurg. 2010;112(3):666-673.

43. Reinhard M, Roth M, Guschlbauer B, et al. Dynamic cerebral autoregulation in acute ischemic stroke assessed from spontaneous blood pressure fluctuations. Stroke. 2005;36(8): 1684-1689.

44. Reinhard M, Wihler C, Roth M, et al. Cerebral autoregulation dynamics in acute ischemic stroke after rtPA thrombolysis. Cerebrovasc Dis. 2008;26(2):147-155.

\section{Disclosures}

Dr. Luft: honoraria from Moleac, and speakers bureau for Bayer and Amgen.

\section{Author Contributions}

Conception and design: all authors. Acquisition of data: Sebök, van Niftrik. Analysis and interpretation of data: Sebök, van Niftrik. Drafting the article: Sebök, van Niftrik, Regli, Fierstra. Critically revising the article: all authors. Reviewed submitted version of manuscript: all authors. Approved the final version of the manuscript on behalf of all authors: Sebök. Statistical analysis: Sebök, van Niftrik. Administrative/technical/material support: Sebök, van Niftrik. Study supervision: Regli, Fierstra.

\section{Correspondence}

Martina Sebök: University Hospital Zurich, Switzerland. martina. seboek@usz.ch. 